\title{
Reutilización de componentes en la producción de MOOC: un caso de estudio
}

\section{Guillermo Castilla Alcaláa, Jose Ignacio Ortiz González ${ }^{\text {b }}$, Alfonso Durán Heras} Escuela Politécnica Superior de la Universidad Carlos III de Madrid, España, acastilla@ing.uc3m.es, bjoortiz@ing.uc3m.es, ${ }^{\text {cduran } @ i n g . u c 3 m . e s ~}$

\section{Resumen}

Los MOOC (Massive Open Online Courses) son un recurso docente potencialmente muy valioso, bien como instrumento en sí o como complemento de la docencia presencial. Uno de sus principales inconvenientes es la dedicación y esfuerzo requerido para el desarrollo de un MOOC de calidad.

El número de MOOC que una institución educativa desarrolla con temáticas similares, personalizados para distintas audiencias y contextos se incrementa cada año. Por tanto, cobra importancia la oportunidad de reutilizar componentes de los MOOC ya desarrollados para disminuir el coste y el tiempo de producción de los nuevos MOOC.

Dado que es un fenómeno relativamente reciente, no es infrecuente que las instituciones, se planteen esta necesidad de reutilización únicamente a posteriori, cuando surgen las necesidades de nuevos MOOC relacionados o de variantes del inicial. Esto les enfrenta a importantes dificultades, que podrían haberse paliado con un diseño orientado desde el principio a la flexibilidad y la reutilización, adoptando metodologías como las propuestas en este trabajo.

Esta orientación a la reutilización puede inspirarse en metodologías desarrolladas en otros ámbitos con ese mismo propósito, como la programación estructurada, la programación modular y más concretamente la programación orientada a objetos.

Los componentes de los MOOC cuyo desarrollo consume más recursos son normalmente los vídeos y los test de evaluación. En este trabajo se revisan buenas prácticas de diseño de estos componentes que facilitan su reutilización de forma eficiente.

Este trabajo presenta el caso de reutilización de diversos componentes de un MOOC de la plataforma edX dedicado a Sistemas de Información, 
inicialmente diseñado para una gran diversidad de participantes, cuyos componentes son reutilizados para desarrollar otros dos cursos virtuales: un SPOC (Small Private Online Course) específico para alumnos de ingeniería y un segundo sobre la toma de decisiones empresariales dirigido a personal de administración y servicios de Naciones Unidas (UN).

Palabras clave: $M O O C$, SPOC, reutilización, metodología, producción.

\section{Introducción: MOOC una tendencia al alza en los procesos de enseñanza}

Los MOOC (Cursos Online Masivos Abiertos) son herramientas docentes innovadoras que permiten a las universidades contribuir a la enseñanza de calidad de la sociedad, sin restricciones espacio temporales. Básicamente, se dice que un MOOC es abierto porque es accesible a cualquier usuario.

Los MOOC marcan tendencia en el mundo del e-learning y la actividad de creación de MOOC por parte de las universidades va en aumento. Cada vez hay más usuarios de este tipo de cursos, y lo que es más importante, el porcentaje de personas que después de realizar un MOOC están dispuestas a volver a participar en este tipo de cursos va en aumento (Callejo-Gallego \& Agudo-Arroyo, 2018).

Los MOOC se diseñan para una audiencia masiva global, pero en muchas ocasiones surge la necesidad de adaptación a contextos específicos, siendo preciso personalizar los MOOC a audiencias determinadas.

Una vez se decide la temática y objetivos del MOOC, el proceso de creación de un MOOC es laborioso, requiriendo gran dedicación y esfuerzo. La elaboración de un MOOC precisa que se planifiquen y diseñen en detalle los distintos componentes que lo constituyen, tales como:

- Vídeos donde se explican los conceptos grabados por el profesor. Estos vídeos son vídeos de corta duración (inferiores a 10-15 minutos) confeccionados con modelos tales como el de busto parlante y/o de pizarra electrónica tipo Khan Academy (Khan Academy Inc, 2019). Una lección está constituida por uno o varios vídeos. Y un conjunto de lecciones constituyen un módulo del MOOC. Las tres fases principales del proceso de elaboración de los vídeos, en términos de consumo de recursos, son: preproducción (planificación y creación del guion), captura (grabación), y procesamiento (postproducción).

- Transcripciones con el guion escrito del vídeo, que contienen el texto escrito de todo lo dicho por el profesor en el vídeo; pueden utilizarse también para el subtitulado. 
- Material de apoyo tal como dibujos y transparencias que se utilizan en el vídeo u otros materiales complementarios (otros vídeos, artículos etc.). Requieren mención especial, por su potencial didáctico pero también por el esfuerzo que requieren, los distintos tipos de animaciones, desde las basadas en gráficos móviles a las creadas con software

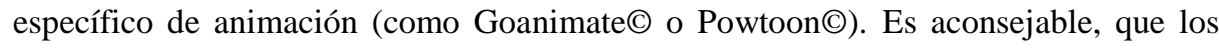
dibujos y transparencias no contenga mucha información y fuercen al alumno a prestar atención a las explicaciones del profesor para entenderlos (Unidad de Tecnología e Innovacion Docente Servicio de Biblioteca Universidad Carlos III de Madrid (UTEID), 2014).

- Actividades de autoevaluación de lecciones (vídeos) o módulos. Para evaluar los vídeos se hacen pequeños test (quizzes) que evalúan la asimilación de los conceptos explicados en la lección. Para los módulos se usa un test que permita valorar el grado de desarrollo y evolución del alumno a lo largo del MOOC. Los quizzes se responden en poco tiempo y en ellos se ofrece varias opciones de respuesta a las preguntas, entre las que se encuentra la respuesta correcta. Los test de módulos son pruebas de evaluación más extensas y complejas que consisten en una serie de preguntas, tareas y/o problemas a resolver, que requieren de mayor análisis y razonamiento para responderlas. Dentro de estos componentes también se pueden incluir ejercicios o asignaciones de proyectos, así como el sistema de corrección utilizado.

La creación de vídeos de calidad requiere el uso de software de edición de vídeo que a menudo es complejo y precisa conocimientos técnicos de grabación de vídeo y de utilización de la línea de tiempo. Recientemente se están desarrollando software y metodologías para facilitar y simplificar el proceso de producción de los vídeos (Bakkay et al., 2019).

La razón por la que los vídeos que constituyen los MOOC son de corta duración (Leton, Durban, D'Auria, \& Lee, 2009) es que el tiempo de atención sostenida de una persona no suele superar los 10-15 minutos.

\section{Reutilización de Componentes: un aspecto clave para la eficiencia}

La personalización de MOOC y las necesidades de adaptación a múltiples entornos precisa el uso de metodologías de producción de contenidos, que al igual que la programación basada en objetos del desarrollo software, minimicen el coste del desarrollo basándose en la reutilización de componentes.

La reutilización de componentes es un elemento clave en la eficiencia de múltiples actividades de los procesos educativos. Cuando aparecieron las plataformas LMS (Learning 
Management System) o VLE (Virtual Learning Environment) tal como Moodle, Claroline, Edoome etc, se definieron estándares como SCORM (Sharable Content Object Reference Model) que permitían la creación de SCO (sharable content objects/objetos de aprendizaje) que pueden reutilizarse en diferentes plataformas y contextos. De este modo, componentes de un MOOC que sigan el estándar SCORM pueden transferirse entre plataformas que siguan dicho estándar.

Mediante el uso de recursos de aprendizaje modulares se mejora la actividad educativa de los LMS de recursos en formatos SCORM. Actualmente se habla del diseño instruccional basado en objetos.

Además de construir componentes reutilizables en otras plataformas, puede considerarse la reutilización de componentes desarrollados para la confección de MOOC derivados. A la hora de grabar el vídeo de la presentación, puede ser conviente segmentarlo y tener en cuenta ciertos aspectos que faciliten la reutilización de los distintos segmentos en futuras adaptaciones del MOOC.

Un caso sería el primer vídeo de la lección 1.1, correspondiente a la presentación del curso donde se incluiría puntos como: presentación del profesorado, objetivos, temario, criterios de evaluación, examen, recursos bibliográficos o videográficos, planificación del curso, calendario/duración, metodología docente, conocimientos requeridos, competencias que adquirirá el alumno, funcionamiento del MOOC, etc.

Este vídeo puede realizarse pensando en la posibilidad de cortar partes del mismo, y que en cualquier momento se pueda cambiar una de las secuencias del vídeo, por ejemplo, con un temario reducido, y que el vídeo pueda tener sentido. Con este enfoque no hay que incluir lista detallada de las partes de la presentación tipo índice, sino que basta con incluir mediante un esquema cronológico o espacial los puntos de la presentación al más alto nivel, con tres componentes estructurales, por ejemplo:

\section{"Presentación" $\rightarrow$ "Programa" $\rightarrow$ "Evaluación"}

De este modo, en la componente "Presentación" se podría incluir la presentación del profesorado, recursos bibliográficos, conocimientos requeridos, competencias a adquirir y Objetivos; en la parte de "Programa" se incluirían la planificación del curso, duración, temario, metodología docente, y en la "Evaluación" cualquier asunto relacionado con pruebas y ejercicios.

Especialmente en vídeos que utilizan, al menos en parte, el enfoque de "busto parlante", no es recomendable incorporar posteriormente nuevos segmentos grabados que incluyan el profesor, dada la práctica imposibilidad de igualar vestuario, peinado etc. Por tanto, es recomendable incluir en la grabación adicional diversas variantes (p.ej., diversos inicios y finales), junto con las mini-pausas orientadas a facilitar el ensamblado de distintos vídeos. 
Sin embargo, un escenario en el que no es recomendable la reutilización de las secuencias es en el vídeo de presentación, que se difunden fuera del contenido del MOOC, bien en la propia página de introducción (o en la página informativa "A cerca de") del curso para inscribirse en el curso o en otras plataformas webs como YouTube, redes sociales o webs de la propia universidad etc, ya que ese vídeo tiene un componente publicitario que ha de adaptarse al público objetivo, por lo que en este caso se trata de un vídeo que ha de personalizarse para cada MOOC (Rajas, Puebla-Martínez, \& Baños, 2018).

\subsection{Ideas para la reutilización de vídeos}

El punto clave para la reutilización de vídeos (lecciones) es que cada vídeo solo tenga un objetivo docente y solo explique un único concepto. Esto conduce a que en un MOOC puedan existir vídeos de duración variable (desde menos de 1 minuto hasta 15 minutos si fuera necesario). Los vídeos han ser cortos y concretos. 
Tabla 1. Recomendaciones para facilitar la reutilización de vídeos

\begin{tabular}{|c|}
\hline Recomendaciones \\
\hline Si se plantea una pregunta a la audiencia, incluir la respuesta en el propio vídeo \\
\hline Si se plantea un ejercicio en el vídeo, incluir la respuesta en el propio vídeo \\
\hline Vídeo completo independiente con el/los objetivos del curso /modulo / lección \\
\hline Si se relaciona el tema con un caso de la vida real, que sea adecuado a la audiencia global \\
\hline Evitar cometarios/referencias relacionando conocimientos previos o lecciones/vídeos anteriores \\
\hline Vídeo independiente para desglosar los puntos que se desarrollan en el curso / módulo / lección \\
\hline Evitar evidenciar una sucesión de contenidos o temáticas \\
\hline Evitar usar expresiones locales o adaptadas a una audiencia concreta. \\
\hline Mantener el mismo ritmo en los vídeos, evitando sorpresas o puntos de giro narrativos. \\
\hline Poner ejemplos y referencias de lo que se está hablando en el vídeo (no de otros) \\
\hline Establecer conclusiones de acuerdo con lo planteado en el inicio del vídeo. \\
\hline $\begin{array}{l}\text { Realizar resúmenes o síntesis de lo abordado en el vídeo ( si es resumen de un conjunto de } \\
\text { vídeos/lección, hacerlo en otro vídeo) }\end{array}$ \\
\hline $\begin{array}{l}\text { Cerrar los vídeos con un mensaje de despedida de "hasta pronto" o "hasta el próximo vídeo" y si se } \\
\text { hacen referencias a los ejercicios asociados al vídeo que alumno debería realizar, separar la secuencia } \\
\text { claramente para poder cortarla. }\end{array}$ \\
\hline Evitar mencionar los puntos que se verán en la siguiente lección o vídeo \\
\hline Evitar cambios de plano en la cámara entre los distintos vídeos \\
\hline $\begin{array}{l}\text { Mantener el formato del material de apoyo (gráficos, diagramas, dibujos, ppt's etc.) constante a lo } \\
\text { largo de todos los vídeos; mismo color de pizarra, mismo tipo de letra, estilo de vestuario del } \\
\text { profesor, mismos entorno, mismo tipo de gráficos y colores. }\end{array}$ \\
\hline Las imágenes que aparecen en cada vídeo se relacionan con el contenido del mismo ( lección) \\
\hline $\begin{array}{l}\text { Utilizar el número mínimo de imágenes y transparencias/diapositivas , sin saturarlos de texto, solo } \\
\text { incluir palabras clave, ideas y conceptos. }\end{array}$ \\
\hline
\end{tabular}

Los vídeos han de realizarse lo más independiente posible sin que unos citen a otros vídeos. Hay que evitar expresiones del tipo "como hemos visto en el vídeo anterior...", "según hemos estudiado en el capítulo...” etc. Además, hay que tratar de mantener el mismo formato en todos los vídeos.

En la Tabla 1 se indican recomendaciones relacionadas con la secuenciación de los vídeos en relación a su estructura narrativa para facilitar la reutilización.

\section{Caso de Estudio}

Se presenta el caso de reutilización del material audiovisual del MOOC "Introducción a los Sistemas de Información Gerencial (SIG): Una guía de supervivencia”. ("Introduction to Management Information Systems (MIS): A Survival Guide”) de la plataforma edX 
dedicado a entender los Sistemas de Información Gerencial y el papel que estos desempeñan en las organizaciones de hoy en día.

Este MOOC se diseñó a lo largo del año 2015, la primera edición fue en 2016, destinado a una audiencia diversa con conocimientos básicos de informática a nivel de usuario y con inquietudes sobre conocimientos de gestión empresarial. El curso se planteó con 6 módulos para una duración de 6 semanas, cuya estructura de módulos se muestra en la Tabla 2.

Cada módulo consta de un máximo de 6 lecciones, cada una de las cuales consta de 1 o 2 vídeos. Cada lección incluye un test tipo quiz con 2 a 4 preguntas. Al final de cada módulo se realiza una prueba de evaluación del módulo más extensa.

A partir de este MOOC inicial surgió la necesidad de crear dos nuevos cursos virtuales: uno en versión SPOC (Curso Online Privado Pequeño-Small Private Online Course) específica para alumnos del European Virtual Exchange (EVE) en ingeniería de la Universidad Carlos III en la plataforma de MOOC de la propia universidad (spoc.uc3m.es), basada en Open edX; y un segundo sobre la toma de decisiones empresariales dirigido a otra audiencia tal como el personal de administración y servicios de la Organización de Naciones Unidas (ONU).

\subsection{Curso virtual EVE}

En el caso del EVE, el ámbito de contenidos a cubrir era análogo al del MOOC original, lo que permitió mantener la estructura básica del curso. Adicionalmente, la similitud de las plataformas (Open edX vs. plataforma edX) permitió la exportación directa de gran parte del contenido.

Fueron necesarias, no obstante, ciertas particularizaciones para adaptar el curso al nuevo ámbito. Una primera diferencia afectaba al público objetivo, restringido, en el caso del EVE, a estudiantes de ingeniería. Una segunda, relacionada, afectaba a la evaluación. Aparte de las implicaciones del cambio en el público objetivo, y a diferencia de los cursos MOOC, el SPOC de EVE conducía a la obtención de créditos formales. La combinación de ambos factores exigió complementar el MOOC original con diversas adiciones. Los cuestionarios sumativos (que repercuten en la evaluación, a diferencia de los formativos, centrados en la autoevaluación) se modificaron para estar basados en librerías de preguntas. De esta forma, a cada alumno se le presentaba una selección al azar de preguntas de la librería, diferente para cada uno, mejorando así la garantía de "originalidad". Esto se complementaba con la introducción de un examen presencial integrado obligatorio; asimismo, los trabajos asignados en el MOOC se complementaban con un proyecto integrado ("Capstone Project"), cuya realización exigía una comprensión en mayor 
profundidad del material presentado, así como recurrir a material adicional que en el MOOC se trataba como opcional.

Adicionalmente, el nuevo entorno y las nuevas reglas exigieron una serie de cambios que, gracias al diseño modular inicial, pudieron compaginarse con la reutilización del material. Así, en parte se plasmaron en el material escrito, tanto el "Syllabus" como las entradillas de texto en html que posicionan y relacionan los vídeos y ejercicios, y que son más fácilmente modificables. En cuanto a los cambios requeridos en los vídeos en sí, la práctica de grabar, desde la primera versión, distintos inicios y finales para cada vídeo permitió minimizar el impacto de los cambios.

Resulta interesante destacar que una parte del trabajo adicional requerido para generar el curso derivado pudo reutilizarse para enriquecer el producto original. Así, el uso de librerías en las preguntas sumativas, si bien no resulta imprescindible en un MOOC no orientado a otorgar créditos (y normalmente los MOOC no lo incluyen), sí supone una mejora útil. Así pues, en este caso, habiendo hecho ya el trabajo, se implantó asimismo en el MOOC abierto.

\subsection{Curso Information Management para la toma de decisiones (UN)}

El objetivo del segundo curso virtual fue hacer ver al alumno la importancia de los sistemas de información para la toma de decisiones en las organizaciones, ya que no son solo el medio para recolectar, procesar y almacenar los datos que genera la organización en sus operaciones, sino que aportan distintas herramientas que facilitan la realización de un análisis de los datos y la toma de decisiones informadas.

En este caso, a diferencia del anterior, el ámbito de contenidos a cubrir era tan sólo un subconjunto del MOOC original, exigiendo no sólo la selección de un subconjunto de los componentes del MOOC original sino adicionalmente la reconstrucción de los enlaces entre ellos, así como cubrir mínimamente los aspectos introducidos en los vídeos no incluidos a los que se hacía referencia en los vídeos incluidos.

En la grabación del primer MOOC se siguieron las recomendaciones indicadas en la Tabla 1, lo que permitió introducir los cambios necesarios en los vídeos originales, y "reenlazarlos" mediante cuatro nuevos "vídeos de posicionamiento", con un tiempo neto total de tan sólo 15 minutos de nuevas grabaciones. También se usaron las entradillas de texto. 
Tabla 2. Estructura de los módulos del MOOC

\begin{tabular}{|c|c|c|c|}
\hline & Videos / Script & Tests & $\begin{array}{l}\text { Examen/ } \\
\text { Proyecto } \\
\text { /Trabaio }\end{array}$ \\
\hline \multicolumn{4}{|l|}{ Módulo 1. SIG. Importancia. Desarrollo o compra } \\
\hline 0 Presentation & 0.0 Bienvenida al curso, introducción y e structura & & \\
\hline \multirow[t]{2}{*}{$\begin{array}{l}\text { 1.1. La invasión de los Sistemas de Información } \\
\text { Gerencial }\end{array}$} & 1.1.1 SIGs: en la frontera entre TI y negocio & Ejercicio 1.1 & Test 1 \\
\hline & 1.1.2 No se puede vivir sin SIGs (ni con) & & \\
\hline \multirow[t]{2}{*}{ 1.2. Adquisición de SIG } & 1.2.1 Hacer o Comprar & Ejercicio 1.2 & \\
\hline & 1.2.2 Subcontratación, deslocalización & & \\
\hline 1.3. Desarrollo de SIG & 1.3.1 Desarrollo de SIG. M etodologías & Ejercicio 1.3 & \\
\hline 1.4. Sistemas abiertos. Código abierto & 1.4.1 Sistemas abiertos. Código abierto & Ejercicio 1.4 & \\
\hline $\begin{array}{l}\text { Módulo 2. La revolución de las comunicaciones - } \\
\text { Sistemas de Información Distribuidos }\end{array}$ & & & Test 2 \\
\hline $\begin{array}{l}\text { 2. 1. Introducción: Sistemas distribuidos e infraestructura } \\
\text { distribuida }\end{array}$ & $\begin{array}{l}\text { 2.1.1 Introducción: Sistemas distribuidos e } \\
\text { infraestructuras distribuidas }\end{array}$ & Ejercicio 2.1 & \\
\hline \multirow[t]{2}{*}{ 2.2. SI distribuido. Cliente-servidor } & 2.2.1 SI distribuido. Cliente-servidor de 2 niveles & Ejercicio 2.2 & \\
\hline & 2.2.2 Cliente-servidor multinivel & & \\
\hline \multirow[t]{2}{*}{$\begin{array}{l}\text { 2.3. In fraestructuras distribuidas. Redes de } \\
\text { telecomunicaciones }\end{array}$} & $\begin{array}{l}\text { 2.3.1 Infraestructuras distrib uidas. Red de área } \\
\text { local (LAN) }\end{array}$ & Eiercicio 2.3 & \\
\hline & $\begin{array}{l}\text { 2.3.2 Infraestructuras distrib uidas. Red de área } \\
\text { amplia (WAN) }\end{array}$ & & \\
\hline \multirow[t]{3}{*}{ 2.4. La Nube } & 2.4.1 Nubes privadas & Eiercicio 2.4 & \\
\hline & 2.4 .2 Nubes públicas & & \\
\hline & $\begin{array}{l}2.4 .3 \text { Bene ficios de nubes privadas, nubes } \\
\text { públicas y nubes híbridas }\end{array}$ & & \\
\hline \multirow[t]{2}{*}{ Módulo 3. Datos, Bases de Datos, Big Data } & & & Test 3 \\
\hline & $\begin{array}{l}\text { 3.1.1 Datos: un ingrediente clave en los SIG } \\
(2017 \text { ESP_3.1_Datos: Un ingrediente clave en } \\
\text { los SIG) }\end{array}$ & Ejercicio 3.1 & \\
\hline $\begin{array}{l}\text { 3.2. Bases de datos, Estructura de la Base de Datos y } \\
\text { Sistemas de Gestión de Bases de Datos (SGBD) }\end{array}$ & $\begin{array}{l}3.2 .1 \text { Bases de datos, estructura de la base de } \\
\text { datos y SGBD }\end{array}$ & Eiercicio 3.2 & \\
\hline \multirow[t]{2}{*}{ 3.3. Modelización de datos. Bases de datos relacionales } & $\begin{array}{l}\text { 3.3.1 Modelización de datos I. SGBD jerárquico y } \\
\text { relacional (2017_ESP_3.3 M odelado de Datos } 1 . \\
\text { SGBD Jerárquico y Relac) }\end{array}$ & Eiercicio 3.3 & \\
\hline & $\begin{array}{l}\text { 3.3.2 Modelización de datos II. Relaciones. Claves } \\
\text { (2017_ESP_3.4 M odelado de Datos II. } \\
\text { Rela ciones. Claves.) }\end{array}$ & & \\
\hline \multirow[t]{2}{*}{ 3.4. Normalización SQL. Selección de SGBD } & $\begin{array}{l}\text { 3.4.1 Redundancia y Normalización. P apel del } \\
\text { SGBD (2017_ESP_3.5_Redundancia y } \\
\text { Normalización. Papel del SGBD) }\end{array}$ & Eiercicio 3.4 & \\
\hline & $\begin{array}{l}\text { 3.4.2 SQL. Selección de SGBD } \\
\text { (2017_ESP_3.6_SQL. Elección del SGBD) }\end{array}$ & & \\
\hline 3.5. Big Data & 3.5.1 Big Data (ESP_3.5.1_Big Data) & Ejercicio 3.5 & \\
\hline $\begin{array}{l}\text { Módulo 4. S istemas de Información Integrados (ERP) } \\
\text { y Procesos de Negocio Integrados }\end{array}$ & & & Test 4 \\
\hline $\begin{array}{l}\text { 4.1. Introducción a los Sistemas Integrad os de } \\
\text { Información (ERP) }\end{array}$ & $\begin{array}{l}\text { 4.1.1 Introducción a los Sistemas Integrados de } \\
\text { Información (ERP) }\end{array}$ & Eiercicio 4.1 & \\
\hline \multirow[t]{2}{*}{ 4.2. Procesos de Negocio integrados y SI integrados } & $\begin{array}{l}\text { 4.2.1 Procesos de Negocio, Subprocesos y } \\
\text { Transacciones de Negocio }\end{array}$ & Ejercicio 4.2 & \\
\hline & $\begin{array}{l}\text { 4.2.2 Procesos de negocio integrados y } \\
\text { multifuncionales y SI integrados }\end{array}$ & & \\
\hline \multirow[t]{3}{*}{ 4.3. Estructura ERP: M ódulos, Base de Datos común } & 4.3.1 M ódulos E RP semi-independientes & Ejercicio 4.3 & \\
\hline & $\begin{array}{l}\text { 4.3.2 Consecuencias de compartir una Base de } \\
\text { Datos Común I }\end{array}$ & & \\
\hline & $\begin{array}{l}\text { 4.3.3 Consecuencias de compartir una Base de } \\
\text { Datos común II }\end{array}$ & & \\
\hline 4.4. Ventajas y desventajas del enfo que ERP & 4.4.1 Ventajas e inconvenientes del en foque ERP & Eiercicio 4.4 & \\
\hline 4.5. Sesión práctica E RP & 4.5.1 Sesión práctica ERP I & Eiercicio 4.5 & \\
\hline \multirow{3}{*}{$\begin{array}{l}\text { Módulo } 5 \text {. Implementación y personalización de E RPs } \\
\text { y otros grandes SIGs. ¿Quién se adapta a quién? } \\
\text { 5.1. Desafíos y enfoques en la aplicación y adaptación de } \\
\text { los principales sistemas de in formación de gestión }\end{array}$} & 4.0 .2 & & Test 5 \\
\hline & $\begin{array}{l}\text { 5.1.1 Desafíos y enfoques en la aplicación y } \\
\text { adaptación de los principales SIGs }\end{array}$ & Ejercicio 5.1 & \\
\hline & 5.1.2 ¿Quién se adapta a quién? Introducción & & \\
\hline \multirow{3}{*}{$\begin{array}{l}\text { 5.2. Adaptación de la práctica actual. Reingeniería de } \\
\text { Procesos de Neqocio (RPN) } \\
\text { 5.3. Adaptación del ERP }\end{array}$} & 5.2.1 Adaptación de la práctica actual. RPN & Eiercicio 5.2 & \\
\hline & 5.3.1 Elección del E RP. Parametrización & Ejercicio 5.3 & \\
\hline & 5.3.2 Personalización basada en la codificación & & \\
\hline \multirow[t]{2}{*}{ 5.4. I mplementación del ERP } & $\begin{array}{l}\text { 5.4.1 Enfoques de implementación de ERP: Big } \\
\text { Bang, Roll out, Modul }\end{array}$ & Ejercicio 5.4 & \\
\hline & $\begin{array}{l}\text { 5.4.2 Desafíos y costos de la implementación del } \\
\text { ERP }\end{array}$ & & \\
\hline \multirow{2}{*}{$\begin{array}{l}\text { 5.5. Post-implementación del ERP } \\
\text { Módulo 6. SIG en la toma de decisiones }\end{array}$} & $\begin{array}{l}\text { 5.5.1 Post-I mplementación de ERP: una historia } \\
\text { interminable }\end{array}$ & Eiercicio 5.1 & \\
\hline & & & Test 6 \\
\hline 6.1. Toma de decisiones & $\begin{array}{l}\text { 6.1.1 Toma de decisiones. Los que lo hacen, los } \\
\text { que deciden }\end{array}$ & Ejercicio 6.1 & \\
\hline $\begin{array}{l}\text { 6.2. Sistemas transaccionales vs. Sistemas de Apoyo a la } \\
\text { Decisión }\end{array}$ & 6.2.1 La pirámide TPS vs. Ia DSS & Eiercicio 6.2 & \\
\hline \multirow[t]{2}{*}{$\begin{array}{l}\text { 6.3. Retos de los sistemas de apoyo a la toma de } \\
\text { decisiones }\end{array}$} & 6.3.1 Obstáculos y enfoques del DSS I & Ejercicio 6.3 & \\
\hline & 6.3.2 Obstáculos y enfoques del DSS II & & \\
\hline 6. 4. DSS, datos no estructurados y Big Data & $\begin{array}{l}\text { 6.4.1 DSS, datos no estructurados y Big Data. } \\
\text { Análisis }\end{array}$ & Ejercicio 6.4 & \\
\hline 6.5. Una palabra & $\begin{array}{l}\text { 6.5.1 Una palabra sobre el futuro: Inteligencia } \\
\text { Artificial, Machine Learninq... }\end{array}$ & Eiercicio 6.5 & \\
\hline 6.6. Despedida & 6.6.1 Despedida & Ejercicio 6.6 & \\
\hline
\end{tabular}

Editorial Universitat Politècnica de València 
Tabla 3. Estructura de los módulos del curso virtual para UN

\begin{tabular}{|c|c|c|c|}
\hline & Videos /Script & Tests & $\begin{array}{l}\text { Examen/ } \\
\text { Proyecto } \\
\text { /Trabajo }\end{array}$ \\
\hline \multicolumn{4}{|l|}{ Módulo 1. SIG. Importancia. Desarrollo o compra } \\
\hline \multirow[t]{2}{*}{0 Irtroducción } & 0.1 Bienvenida al curso, introducción y estructura & & \\
\hline & 0.0 Introducción Conocimientos base TIC & & \\
\hline $\begin{array}{l}\text { 1.1. La irvasión de los Sistemas de Información } \\
\text { Gerencial }\end{array}$ & 1.1.1 No se puede vivir sin SIGs (ni con) & Ejercicio 1.1 & Test 1 \\
\hline 1.2. Datos: un ingre dierte dave en un SIG & $\begin{array}{l}\text { 1.2 1 Datos un ingredierte dave en los SIG } \\
(2017 \text { ESP_3.1_Datos: Un ingrediente clave en } \\
\text { los SIG) }\end{array}$ & Ejercicio 1.2 & \\
\hline $\begin{array}{l}\text { 1.3. Bases de datos, Estructura de la Base de Datos y } \\
\text { Sistemas de Gestión de Bases de Datos (SGBD) }\end{array}$ & $\begin{array}{l}\text { 1.3.1 Bases de datos, estructura de la base de } \\
\text { datos y SGBD }\end{array}$ & Eiercicio 1.3 & \\
\hline 1.4. Modelización de datos. Bases de datos relacionales & $\begin{array}{l}\text { 1.4.1 M odelización de datos I. SGBD jerárquico y } \\
\text { relacional (2017_ESP_3.3 M odelado de Datos I. } \\
\text { SGBD Jerárquico y Relac) }\end{array}$ & Ejercicio 1.4 & \\
\hline & $\begin{array}{l}\text { 1.4.2 M odelización de datos II. Relaciones. Claves } \\
\text { (2017_ESP_3.4 M odelado de Datos II. } \\
\text { Relaciones. Claves.) }\end{array}$ & Ejercicio 1.5 & \\
\hline 1.5. Biq Data & 1.5.1 Biq Data (ESP 3.5.1 Biq Data) & Ejercicio 1.6 & \\
\hline Módulo 2. SIG en la toma de decisiones & & & Test 2 \\
\hline 0 Irtroducción & 2.0 Video Contextual & & \\
\hline 2.1. Toma de decisiones & $\begin{array}{l}\text { 2.1.1 Toma de decisiones. Los que lo hacen, los } \\
\text { que deciden }\end{array}$ & Ejercicio 6.1 & \\
\hline $\begin{array}{l}\text { 2.2. Sistemas transaccionales vs. Sistemas de Apoyo a la } \\
\text { Decisión }\end{array}$ & 2.2.1 La pirámide TPS vs. la DSS & Ejercicio 6.2 & \\
\hline $\begin{array}{l}\text { 2.3. Retos de los sistemas de apoyo a la toma de } \\
\text { decisiones }\end{array}$ & 2.3.1 O bstáculos y enfoques del DSS I & Ejercicio 6.3 & \\
\hline & 2.3.2 O bstáculos y enfoques del DSS II & & \\
\hline 2.4. DSS, datos no estrudurados y Biq Data & $\begin{array}{l}\text { 2.4.1 DSS, datos no estrudurados y Big Data. } \\
\text { Análisis }\end{array}$ & Ejercicio 6.4 & \\
\hline 2.5 Despedida & 2.5.1 Despedida & & \\
\hline
\end{tabular}

Muchos de los componentes del primer MOOC fueron reutilizados, ocasionalmente con cambios menores, para desarrollar los nuevos cursos. En concreto, se utilizó un vídeo del módulo 1, cinco del módulo 3 y cinco del módulo 6, quedando estructurado en una introducción, dos módulos unidos por un nuevo vídeo para poner en contexto al alumno (vídeo contextual) y un vídeo de despedida. En la Tabla 3 se muestran sombreados los nuevos vídeos que se han grabado para realizar este curso virtual.

Se grabaron dos nuevos vídeos para la introducción 4 y 7 minutos, el vídeo contextual que se corresponde con la introducción al segundo módulo (5 min) y el de despedida (2 min).

Los quizzes fueron los correspondientes a los vídeos del primer MOOC, y los cuestionarios de los módulos fueron una selección de preguntas de la librería de preguntas correspondientes a los módulos 3 y 6 del MOOC original. En este caso, la mayor diferencia entre las plataformas (edX vs. Moodle) dificultó el trasvase automatizado de los test de evaluación, lo que llevó al uso de cuestionarios no basados en librerías.

La carga de trabajo que supuso la producción del curso virtual destinado a Naciones Unidas puede estimarse en menos del $10 \%$ de la carga productiva del primer MOOC. 


\section{Conclusiones}

Se han recopilado un conjunto de recomendaciones que facilitan la reutilización de los vídeos, con lo que se incrementa la eficiencia del proceso de producción de MOOC. Se ha mostrado un caso que muestra que la reutilización de componentes de un MOOC para producir otros cursos virtuales adaptados a otra audiencia y otros objetivos docentes.

\section{Referencias}

Bakkay, M. C., Pizenberg, M., Carlier, A., Balavoine, E., Morin, G., \& Charvillat, V. (2019). Protocols and software for simplified educational video capture and editing. Journal of Computers in Education, 6(2), 257-276. https://doi.org/10.1007/s40692-019-00136-6

Callejo-Gallego, J., \& Agudo-Arroyo, Y. (2018). MOOC: valoración de un futuro. Revista Iberoamericana de Educación a Distancia, 21, 219-241. https://doi.org/http://dx.doi.org/10.5944/ried.21.2.20930

Khan Academy Inc. (2019). Khan Academy. Recuperado de https://es.khanacademy.org/

Leton, E., Durban, M., D’Auria, B., \& Lee, D. J. (2009). Self learning mini-vídeos through Internet and mobile telephones: a help to the student in the Bologna process. EDULEARN09 Proceedings, (July), 4277-4283. Recuperado de http://library.iated.org/view/LETON2009SEL

Rajas, M., Puebla-Martínez, B., \& Baños, M. (2018). Formatos audiovisuales emergentes para MOOCs: diseño informativo, educativo y publicitario/ Emerging audiovisual formats for MOOCs: Informative, educative and advertising design. El profesional de la información, 27(2), 312-321. https://doi.org/10.3145/epi.2018.mar.09

Unidad de Tecnología e Innovacion Docente Servicio de Biblioteca Universidad Carlos III de Madrid (UTEID). (2014). Guía metodológica para la planificación, diseño e impartición de MOOCs y SPOCs. Recuperado de docubib.uc3m.es/MOOCS/Guia-metodologica-MOOC-Wimba/ 